\title{
Will hospital based short stay assessment units improve efficiency of paediatric services?
}

\author{
M.P. Senanayake ${ }^{1}$, TUN de Silva ${ }^{2}$, D.C. Weerasuriya ${ }^{3}$
}

Sri Lanka Journal of Child Health, 2007; 36: 57-9

(Keywords: hospital admissions, assessment units, paediatric services)

\section{Introduction}

Hospital based short stay observation units staffed by trained medical and nursing personnel have been documented to improve efficiency of paediatric services $^{1,2,3,4}$. Reducing patient admissions will decrease midnight occupancy, night time nursing work load and health care costs and preserve the available resources for indoor paediatric patients. Length of hospital stay is related to psychological problems that follow hospitalization of children and is another reason for keeping acute paediatric admissions to a minimum ${ }^{5}$. At the Lady Ridgeway Hospital for Children, Colombo (LRH) outdoor nebulisation is provided by the Emergency Treatment Unit of the out patients department but assessment or observation units as an alternative to acute admissions is currently available only to patients with diarrhoea, suspected dengue fever, head injuries or those needing minor surgical procedures. General paediatric admissions lack this facility.

\section{Objective}

To conduct an audit of acute medical admissions sent home within 24 hours.

\section{Method}

A prospective observational study was carried out in all six medical units in LRH. The study population comprised all paediatric admissions discharged home within 24 hours between $17^{\text {th }}$ April and $16^{\text {th }}$ May, 2006. Those transferred to other hospitals for further care were not included. The main outcome measures of admission and discharge rates, reasons for admission, medical diagnoses, types of referrals, by whom the decision to admit was made,

\footnotetext{
${ }^{1}$ Professor of Paediatrics, Faculty of Medicine, Colombo. ${ }^{2}$ Senior Registrar in Paediatrics, ${ }^{3}$ Registrar in Paediatrics, Professorial Unit, Lady Ridgeway Hospital, Colombo.
}

(Received on 17 August 2006. Accepted on 02 September 2006). parental perception on appropriateness of admission and decision to discharge were gathered from ward registers, clinical notes and discharge summaries written in diagnosis cards and from interviewing parents using a structured interviewer based questionnaire. Patients who returned to hospital within 72 hours following such discharge were investigated further.

\section{Results}

During the one month period in which this audit was carried out, 2822 admissions were made to the six medical wards and $657(23.2 \%)$ patients were sent home within 24 hours, with little or no intervention. One hundred and seventy five $(27 \%)$ of these discharges were admitted for scheduled short-term treatment such as blood transfusions (97), injectable medications (immunoglobulins, long acting hormone therapy etc.) (29) or specialized investigations (46).

Three patients were admitted for reassessment of a previously followed up condition. The remaining 482 (73\%) patients, who left within 24 hours, had been admitted with acute problems. They comprised $17 \%$ of total admissions and the diagnostic spectrum included upper or lower respiratory tract infections with or without wheezing in 197 (41\%), undifferentiated fevers with or without febrile convulsions in $161(33 \%)$ and breakthrough epileptic seizures $(<1 \%)$. A further $124(25.7 \%)$ were admitted with symptoms that settled within a day but with no specific diagnosis being made. Vomiting, abdominal pain, excessive crying and pruritus due to skin allergies were the predominant complaints in these patients.

Ninety five percent of the 482 patients discharged within a day were self-referrals. The decision to admit had been made by doctors in the out patients department in $404(83.8 \%)$. Parental anxiety and request for admission accounted for 56 (11.6\%), general practitioner referrals for 20 (4\%) and 
consultant referral for $2(0.4 \%)$. One patient had been transferred from another hospital.

Parental perception of the need for hospital admission was as follows: $286(59.3 \%)$ claimed they had preferred ambulatory care to hospitalization but were advised that admission was necessary. Regarding the appropriateness of the discharge and the ability to manage the child at home, 404 (83\%) agreed with the decision and were confident of caring for the child at home. The remainder had not disagreed with the decision but had concerns regarding the child's condition. Only $21.9 \%$ of parents said their opinion had been taken or considered regarding the discharge.

We found that 3\% of those discharged within a day returned within 72 hours, with persistence $(64 \%)$ or recurrence $(29 \%)$ of symptoms. None had serious consequences.

\section{Discussion}

The philosophy to admit only those children whose needs cannot be met without admission to hospital is increasingly adhered to worldwide ${ }^{6}$. Day assessment or observation wards are being created in many paediatric units. We attempted to assess the need for this service reorganization at the largest children's hospital in Sri Lanka. Although a tertiary referral hospital, LRH accepts walk-in patients and 95\% of those who had a rapid turn over were self-referrals. We therefore presume that this data is representative of paediatric admissions to secondary and primary care hospitals in this country.

The existing facilities at the LRH indicate that the Diarrhoea Observation Unit reduces hospital admissions of patients with diarrhoea, by approximately $89 \%{ }^{7}$. We addressed the need for short stay facilities in general paediatric wards.

Our audit found that nearly one in four acute paediatric admissions are discharged within 24 hours. Appropriateness of admissions was not the objective of this paper and given the rapid nature of progression and recovery of acute paediatric conditions the decision to admit cannot be faulted. We found $27 \%$ of those discharged within a day were admitted for a planned intervention that required only a short stay. However, they too went through the same process of admission, paperwork, clerking, accommodation and discharge. A separate short stay facility would have streamlined the entire process form admission to discharge, reduced patient inconvenience and avoided unnecessary administrative, nursing and clinical staff workload.
The published evidence is that short stay paediatric assessment units utilize nursing skills and rosters better and help increase the confidence of parents in the care of their sick children ${ }^{4}$. Easing the problem of overcrowding in wards and preserving indoor resources for the more deserving patients are other important benefits.

Disadvantages of the rapid turnover should be borne in mind and too early or inappropriate discharge prevented. Unscheduled return visits are a means of evaluating the quality of care of short stay units. We found that $3 \%$ of patients returned within 72 hours, a cut off utilized in other studies on the premise that it reflects on causes related to the original complaint ${ }^{8}$. In the absence of an inter-hospital computerized database we were unable to gather information on patients who may have got admitted to neighbouring hospitals.

We recommend assessment or short stay observation units to be created to cater to children whose complaints are likely to resolve in a short period of time. Further research on logistics and cost are needed. In this culture of self-referral and expectation of hospitalization parental perception is important. Discussion with parents is essential for successful implementation of such units. This does not appear to be taking place adequately at present.

This audit has service implications. Implementing hospital based short stay assessment units would improve efficiency of paedaitric services in this country.

\section{Acknowledgements}

We thank Prof. S P Lamabadusuriya, Drs. D H Karunatileke, B J C Perera, Shanthamali de Silva, Jeevani Sarathchandra and Duminda Pathirna, for allowing us to study patients under their care.

\section{References}

1. Browne GJ, Penna A. Short stay facilities: the future of efficient paediatric emergency services, Archives of Diseases in Children 1996; 74:30913.

2. Beverley DW, Ball RJ, Smith RA et al. Planning the future: the experience of implementing a children's day assessment unit in a district general hospital. Archives of Diseases in Children 1997; 77: 287-93. 
3. Meates M. Ambulatory paediatrics - making a difference. Archives of Diseases in Children 1997; 76:468-76

4. Ogilvie D. Hospital based alternatives to acute paediatric admission: a systematic review. Archives of Diseases in Children 2005; 90(2):138-42.

5. Rennick JE, Johnston CC, Dougherty G, Platt R, Ritchie JA. Children's psychological responses after critical illness and exposure to invasive technology. Journal of Developmental and Behavioral Pediatrics. 2002; 23(3):133-44.

6. Hill AM. Trends in paediatric medical admissions. British Medical Journal 1989; 298: 1479-83.
7. Admission Register 2005, Diarrhoea Treatment Unit. Lady Ridgeway Hospital for Children, Colombo.

8. Kreger BE, Restuccia JD. Assessing the need to hospitalize children: paediatric appropriateness evaluation protocol. Pediatrics 1989; 84:242-7.

9. Werneke U, Smith H, Smith IJ, Taylor J, MacFaul R. Validation of the paediatric appropriateness evaluation protocol in British practice. Archives of Diseases in Children 1997; 77: 294-8.

10. Lal K, Kibirige MS. Unscheduled return visits within 72 hours to an assessment unit. Archives of Diseases in Children 1999; 80:455-8. 